\title{
Overexpression of Potassium Channel RNA: In vivo Development Rescues Neurons from Suppression of Morphological Differentiation in vitro
}

\author{
Susan M. Jones, Alison D. Hofmann, Janet L. Lieber, and Angeles B. Ribera \\ Department of Physiology, University of Colorado Health Sciences Center, Denver, Colorado 80262
}

Neuronal differentiation often proceeds differently in vitro than it does in vivo. Previous work demonstrated that overexpression of potassium channel RNA reduces the number of morphologically identifiable neurons that appear in cultures prepared from neural plate stage (17-1/2 hr) embryos (Jones and Ribera, 1994). Here, we report that morphological differentiation of neurons in situ is only slightly affected by overexpression of potassium channels. Endogenous factors appear to compensate for the effect of channel overexpression. Consistent with this view, when cultures are prepared from older neural tube embryos (22-24 $\mathrm{hr}$ ), more neurons containing excess potassium channel RNA differentiate morphologically in vitro. Exposure in situ to a rapid intracellular calcium chelator, but not to tetrodotoxin, $\omega$-conotoxin or a slow calcium chelator, prevents the compensation provided by extended development in vivo. Typically, RNA overexpression is limited to half of the embryo in order to provide an internal control. However, when potassium channel RNA is overexpressed throughout the embryo, few neurons differentiate morphologically in vitro, even if cultures are prepared from older neural tube embryos. Thus, recovery is possible if a minimum of $5 \mathrm{hr}$ of further development in vivo is allowed under conditions in which rapid elevations of intracellular calcium are permitted and half of the nervous system has normal levels of potassium channel RNA. These results suggest that different or additional mechanisms operate in situ than in vitro to promote morphological differentiation of neurons.

[Key words: neuronal differentiation, RNA overexpression, delayed rectifier potassium current, Shaker-like potassium channels, Xenopus embryo, morphologlcal differentiation]

Excitability and neurites are acquired by developing Xenopus laevis primary spinal neurons shortly after their last round of DNA synthesis (Baccaglini and Spitzer, 1977; Lamborghini, 1980; Hartenstein, 1989). These neurons fire action potentials in

\footnotetext{
Received Aug. 8, 1994; revised Sept. 23, 1994; accepted Oct. 21, 1994.

We thank Dr. Ed Callaway for comments on the manuscript and Drs. Paul Fuchs and Michael Zidanic for a gift of $\omega$-conotoxin GVIA. Drs. Xiaonan Gu and Nick Spitzer generously provided information prior to publication. This work was supported by NRSA postdoctoral fellowship NS09237 (S.M.J.) and NIH Grants RO1-NS25217 and KO4-NS01531 (A.B.R.).

Correspondence should be addressed to Angeles B. Ribera, Department of Physiology C-240, University of Colorado Health Sciences Center, Denver CO 80262.

Copyright (C) 1995 Society for Neuroscience $\cdot 0270-6474 / 95 / 152867-08 \$ 05.00 / 0$
}

situ approximately $8 \mathrm{hr}$ after they have become postmitotic but $2 \mathrm{hr}$ before neurite outgrowth first occurs (Baccaglini and Spitzer, 1977; Taylor and Roberts, 1983; Hartenstein, 1993). In vitro, neurons display a parallel pattern and initiate neurite outgrowth $6 \mathrm{hr}$ after plating when they are cultured from early neurula embryos (Spitzer and Lamborghini, 1976). At this time, action potentials are elicited from every cell with a neuronal morphology, indicating that neurite extension in vitro does not precede acquisition of excitability. Thus, the initial appearances of electrical excitability and neuronal morphology occur at similar times in vivo and in vitro.

Electrical activity has the opportunity to influence initial aspects of morphological differentiation, since impulses are recorded before neurites are present. The impulse in immature neurons is of long duration and calcium dependent (Spitzer and Lamborghini, 1976; Baccaglini and Spitzer, 1977). Action potential generation occurs spontaneously and leads to transient elevations in intracellular calcium, which appear to be required for subsequent elaboration of neuronal phenotypes (Holliday and Spitzer, 1990; Gu et al., 1994). Potassium channel RNA overexpression leads to an increase in potassium current density, which reduces the duration of action potentials (Jones and Ribera, 1994). Overexpression of potassium channel RNA leads to a decrease in the number of morphologically differentiated neurons that appear in vitro (Jones and Ribera, 1994). These findings suggest a link between impulse generation and morphological differentiation.

Since the durations of action potentials are shortened, potassium channel RNA overexpression is expected to reduce the substantial calcium influx that occurs during early impulse generation. Intracellular calcium levels have been shown to influence process outgrowth (Anglister et al., 1982; Cohan et al., 1987; Mattson and Kater, 1987; Cohan, 1992; Davenport and Kater, 1992) as well as neuronal survival (Collins et al., 1991). By reducing calcium influx, potassium channel RNA overexpression could lead to a decrease in the number of morphologically identifiable neurons by promoting cell death and/or inhibiting neurite initiation. Alternatively, since potassium channel activity is correlated with the cell cycle in some cells, such as oocytes (Day et al., 1993) and lymphocytes (Choquet et al., 1987; Amigorena et al., 1990), the reduction in morphologically differentiated neuron number may reflect a delay in the time at which neuronal precursors become postmitotic. These possibilities are examined in the studies reported here in which cultures are prepared from older embryos. In addition, given the similar time course of neuronal morphological differentiation in vivo and in vitro, in situ development of the nervous systems of em- 
bryos with overexpressed levels of potassium channel RNA was examined. The results suggest that additional factors and/or cellcell interactions, some of which may require elevation of intracellular calcium, are present in vivo and promote neurite initiation.

\section{Materials and Methods}

Animals and two-rell stage embryo injections. Xenopus embryos were produced by in vitro fertilization (Moon and Christian, 1989) and staged according to Nieuwkoop and Faber (1967). XShal RNA (80 pg/nl; 5$10 \mathrm{nl}$ ) was co-injected with rhodamine-conjugated dextran (Molecular Probes, Eugene, OR) into one cell of a two-cell embryo as described previously (Jones and Ribera, 1994). For some immunocytochemical experiments, fluorescein-conjugated dextran (Molecular Probes) and $\beta$-galactosidase RNA $(80 \mathrm{pg} / \mathrm{nl})$ were co-injected into the other blastomere to provide an internal control. To produce XShal RNA overexpression in the entire embryo, XShal KNA and rhodamine-conjugated dextran were co-injected into one cell, and XShal RNA was co-injected with fluorescein-conjugated dextran into the other cell. Control embryos for these latter experiments consisted of sibling embryos that were similarly injected in both cells but with $\beta$-galactosidase RNA and dextrans.

Immunocytochemistry. Immunocytochemical analyses were done to examine morphological differentiation of primary sensory neurons (Rohon-Beard cells) using the HNK-1 monoclonal antibody, which recognizes an epitope first expressed in morphologically differentiating Xenopus Rohon-Beard neurons at St. 22 (Nordlander, 1989). The whole-mount immunoperoxidase procedure of Klymkowsky and Hanken (1991) was followed with minor modifications. Embryos were sorted at Sts. 18-19, according to the side of thodamine-conjugated dextran injection that was evident as a pink color imparted by the fluorophore. At Sts. 22-24, embryos were fixed in either $2 \%$ paraformaldehyde and $0.2 \%$ glutaraldehyde or Dent's fixative ( $80 \%$ methanol, $20 \%$ DMSO). Incubations in primary and secondary antibodies were carried out overnight at $4^{\circ} \mathrm{C}$ in Tris-buffered saline (TBS) containing $20 \%$ heat-inactivated horse serum and either $0.1 \%$ Triton X-100 or $5 \%$ DMSO for aldehyde or Dent's fixed embryos, respectively. Primary antibody was anti-HNK-1 (American Type Culture Collection, Rockville, MD) used at a 1:50 dilution; secondary antibody was peroxidase-labeled horse anti-mouse IgG (Vector Laboratories, Burlingame, CA) used at a 1:100 dilution. Overnight antibody incubations were followed by five $1 \mathrm{hr}$ rinses in TBS at room temperature. Reaction with $0.5 \mathrm{mg} / \mathrm{ml}$ diaminobenzidine and $0.03 \%$ hydrogen peroxide for $\sim 20$ min permitted detection of antibody binding. Embryos were then dehydrated and cleared with benzyl alcohol/benzyl benzoate (1:2). In order to verify that only embryos ranging between Sts. 22 and 24 were analyzed, criteria of a minimum and maximum of 9 and 16 somites, respectively, were established (Nieuwkoop and Faber, 1967). In some cases, camera lucida drawings were made to count the number of HNK-labeled somata on each side of the neural tube. The number of HNK-labeled peripheral projections as well as the number of individual neurites in each projection were counted. Data were normalized by dividing the number of projections or neurites by the number of segregated segments in the embryo. Within an embryo, projections per segment on each side were compared permitting the use of paired $t$ test statistical analyses; $p$ values $<0.05$ are indicated.

Cell culture. Dissociated cell cultures of neural plate (St. 15), neural groove (St. 18), or neural tube (Sts. 20-22) embryos were prepared by standard methods (Spitzer and Lamborghini, 1976; Blair, 1983; Ribera and Spitzer, 1989). Morphologically differentiated neurons were counted under phase contrast optics and scored for the presence of fluorescent tracer under epiffuorescence illumination. For experiments in which one blastomere of a two-cell embryo was co-injected with XShal RNA and rhodamine-conjugated dextran, the data are expressed as the ratio of fluorescent to nonfluorescent morphologically identifiable neurons in a single culture (differentiation index; Jones and Ribera, 1994). Statistical comparisons of mean differentiation indexes were performed with nonparametric Mann-Whitney two-tail $t$ tests; $p$ values $<0.05$ are indicated. When RNA and either rhodamine or fluorescein-conjugated dextran were co-injected into both blastomeres, absolute numbers of morphologically identifiable neurons derived from each side were scored. In this case, statistical differences between means were determined with the Student's two-tail $t$ test; $p$ values $<0.05$ are indicated.

Loading of cells in culture with either dimethyl-1, 2 bis(2-amino- phenoxy)ethane- $N, N, N^{\prime}, N^{\prime}$-tetraacetic acid-acetomethoxyl ester (DMBAPTA-AM; $K_{d}$ for $\mathrm{Ca}^{2+}$ of $40 \mathrm{nM}$; Molecular Probes) or ethylene glycol-bis( $\beta$-aminoethylether) $N, N, N^{\prime}, N^{\prime}$-tetraacetic acid-acetomethoxyl ester (EGTA-AM; Molecular Probes) was achieved by a $1 \mathrm{hr}$ incubation in the presence of the indicated concentration of the AM form of the chelator; loading of cells was begun either 1 or $12 \mathrm{hr}$ after plating. These modified calcium buffers are lipophilic and enter cells but are active only when deesterified by intracellular enzymes (Tsien, 1981; Robitaille et al., 1993). Stocks of DM-BAPTA-AM (10 nM) or EGTAAM were prepared in dimethylsulfoxide (DMSO) and frozen in $50 \mu \mathrm{l}$ aliquots. On the day of use, stocks were diluted into culture medium and added to cultures. The final concentration of DMSO added to cultures was $<0.05 \%$; this amount of DMSO did not affect morphological differentiation. Using the calcium indicator dye Fura-2, Drs. Xiaonan $\mathrm{Gu}$ and Nicholas Spitzer have found that loading of neurons in culture with BAPTA-AM suppresses intracellular calcium elevations for periods greater than $12 \mathrm{hr}$ (Gu and Spitzer, personal communication, 1994).

In order to expose neurons in situ to tetrodotoxin (TTX; Calbiochem, La Jolla, CA), $10 \mathrm{nl}$ of a $10 \mathrm{~mm}$ stock solution was injected into the archenteron of St. 17-18 embryos. Cultures were prepared from these embryos when they reached St. 22. Assuming an embryo extracellular space volume of $100 \mu \mathrm{l}$, the expected final concentration of TTX would be $1 \mu \mathrm{M}$. To verify that a blocking concentration of TTX was achieved, embryos were similarly injected with TTX at Sts. 26-27 when a strong swimming response is evoked by touching the embryo. Within $30-45$ min, motor responses could not be elicited: recovery required a minimum of $12 \mathrm{hr}$. In preliminary experiments, the neural groove and plate of St. 18 embryos were separated from endoderm and incubated as an explant in the presence or absence of $1 \mu \mathrm{M}$ TTX for $\sim 4 \mathrm{hr}$ until control sibling embryos reached St. 22 . At this time, the explants were dissociated into single cells and cultures were prepared as described above. Exposure to TTX did not affect the differentiation index. However, under these conditions, there was little or no recovery in the differentiation index for untreated (as well as TTX-treated) explants cultured until the equivalent of St. 22 and, thus, this protocol was not used further.

In situ exposure to DM-BAPTA-AM or EGTA-AM was achieved by injecting $10-25 \mathrm{nl}$ boluses of a $10 \mathrm{~mm}$ stock solution prepared in DMSO into the archenteron of St. 18 embryos: similar injections of DMSO were without effect on either embryo survival or subsequent morphological differentiation of neurons in vitro. Given the assumption of an extracellular space volume of $100 \mu \mathrm{l}$, the final concentrations of BAPTA-AM and EGTA-AM expected were 1 and $5 \mu \mathrm{M}$, respectively. To verify that a blocking concentration of BAPTA was achieved, embryos were similarly injected with the chelators at Sts. $26-27$ as described above. Injection of BAPTA stock led to an inability to evoke a swimming response; the strongest response evoked was a slow body curl. The effect persisted for a minimum of $6 \mathrm{hr}$. Injection of DMSO or EGTA stock into St. 26 embryos did not block motor responses.

In situ exposure to $\omega$-conotoxin GVIA (Sigma Chemical Co., St. Louis, MO) was achieved by repeated injections (10 20) over a $15 \mathrm{~min}$ period of $25 \mathrm{nl}$ of a $100 \mu \mathrm{M}$ stock of the blocker. Final concentrations of $0.25-0.50 \mu \mathrm{M} \omega$-conotoxin GVIA were expected. Similar injection protocol applied to St. 26 embryos resulted in paralysis within 20 min, which was sustained for a minimum of $12 \mathrm{hr}$.

Biophysical analyses. Tight seal whole-cell recordings (Hamill et al., 1981) were performed as described previously (Jones and Ribera, 1994). Glass electrodes were pulled from borosilicate glass (Drummond Scientific $\mathrm{Co}$., Broomall, PA) and filled with $100 \mathrm{~mm} \mathrm{KCl}, 10 \mathrm{~mm}$ EGTA, $10 \mathrm{mM}$ HEPES, pH 7.4. Electrode resistances ranged between 2 and 4 $\mathrm{M} \Omega$ For recording of action potentials in current clamp mode, the bath solution contained $125 \mathrm{~mm} \mathrm{NaCl}, 3 \mathrm{~mm} \mathrm{KCl}, 10 \mathrm{~mm} \mathrm{CaCl}, 5 \mathrm{~mm}$ HEPES, $\mathrm{pH}$ 7.4. For voltage clamp recording of potassium currents, the bath solution consisted of $80 \mathrm{~mm} \mathrm{NaCl}, 3 \mathrm{mM} \mathrm{KCl}, 5 \mathrm{~mm} \mathrm{MgCl}, 10$ $\mathrm{mm} \mathrm{CoCl}_{2}, 5 \mathrm{~mm}$ HEPES, $1 \mu \mathrm{M}$ TTX, pH 7.4. Neurons with short processes $(<50 \mu \mathrm{M})$ were selected for recording to avoid space clamp problems. Currents were sampled at $100 \mu \mathrm{sec}$. Leak subtraction was accomplished using a modified P/4 protocol (pCLAMP, Axon Instruments). The membrane potential was held at $-80 \mathrm{mV}$ and stepped to depolarized voltages ranging from -45 to $+45 \mathrm{mV}$. Outward current is expressed as current density, corrected for cell size. Cell size was estimated by determining cell capacitance from the capacitative transient, and assuming $1 \mu \mathrm{F} / \mathrm{cm}^{2}$ (Sakmann and Neher, 1983). Series resistancc was either compensated electronically $(80-100 \%)$ or mathematically us- 

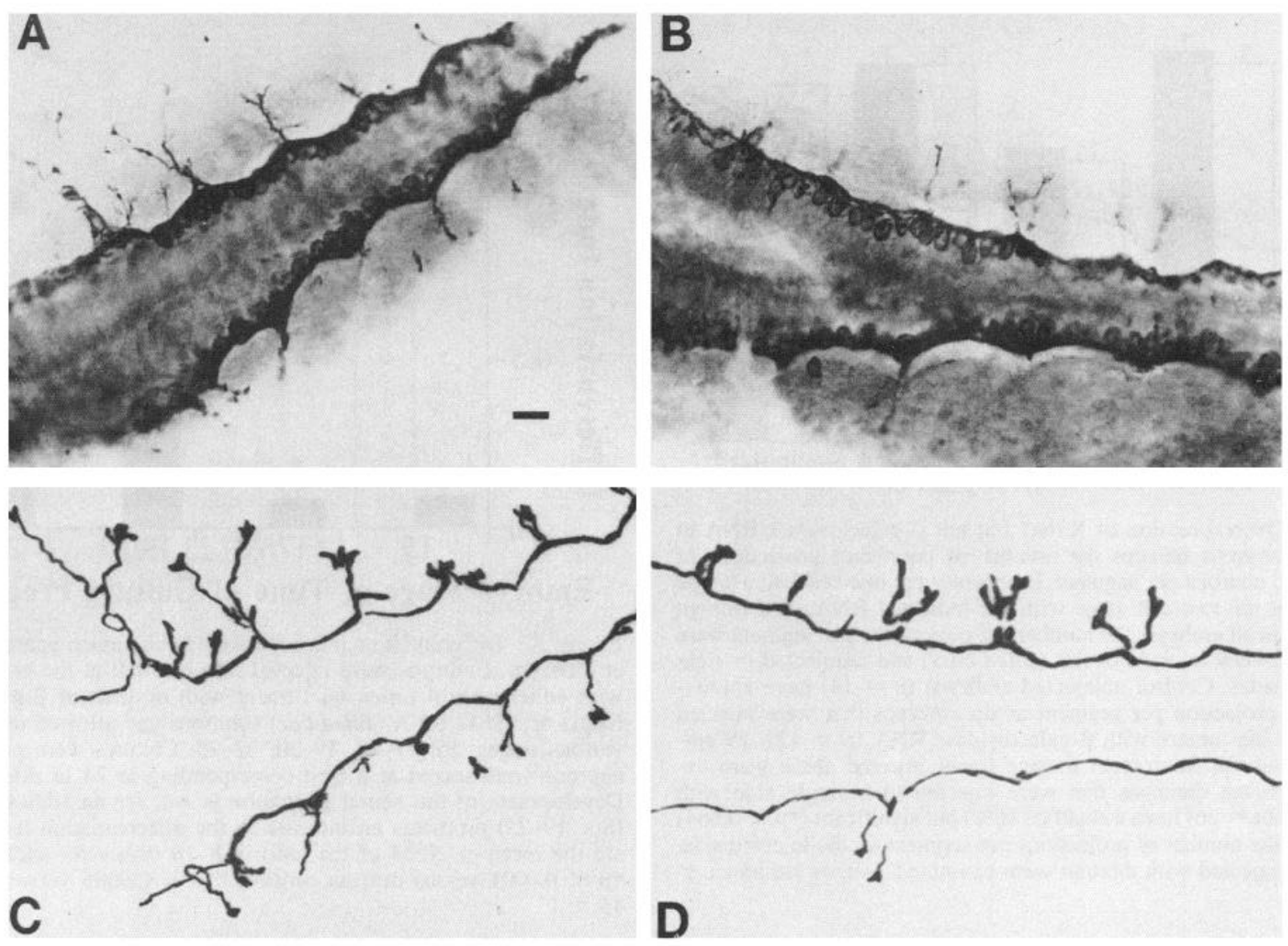

Figure 1. Overexpression of XSha1 RNA reduces the number of peripheral projections of Rohon-Beard neurons in situ. A, Dorsal view of an embryo that was injected at the two-cell stage with rhodamine-conjugated dextran into a single blastomere. Embryos were examined at the wholemount level following indirect immunocytochemistry with HNK-1 antibody, which labels Rohon-Beard neurons and processes in 1-d-old embryos (Nordlander, 1989). The injected side is the left half of the embryo; rostral is to the left. Scale bar, $50 \mu \mathrm{m}$. B, Dorsal view of an embryo that was injected at the two-cell stage with rhodamine-conjugated dextran and XShal RNA into a single blastomere. The injected side is the left half of the embryo; rostral is to the left. $C$ and $D$, Camera lucida drawing of the HNK-1 labeling pattern of $A$ and $B$ shows the individual neurites contained within each projection as revealed by focusing on several different planes.

ing the voltage error introduced by the series resistance (Jones and Ribera, 1994).

\section{Results}

\section{Morphological differentiation in situ}

Overexpression of potassium channel RNA leads to a reduction in the number of morphologically differentiated neurons that appear in culture (Jones and Ribera, 1994). We examined, with whole-mount immunocytochemical analysis, whether similar effects are observed in situ. Since Rohon-Beard cells are primary neurons that differentiate morphologically in culture, this population of neurons was chosen for study in vivo. At the wholemount level in $1 \mathrm{~d}$ embryos, $\mathrm{HNK}-1$ immunoreactivity is evident in Rohon-Beard somata and processes within the dorsolateral fasciculus of the neural tube (Fig. 1; Nordlander, 1989; see Materials and Methods); at this stage, projections of Rohon-Beard neurons are the major contributors of the dorsolateral fasciculus (Dr. Ruth Nordlander, personal communication). In addition, peripheral processes of Rohon-Beard neurons are labeled.

The number of Rohon-Beard peripheral projections was examined to determine whether overexpression of XShal RNA affected this aspect of morphological differentiation (Fig. 2). Embryos were injected at the first cleavage stage into one cell with potassium channel RNA and a lineage tracer. Embryos were allowed to develop until $\sim 1 \mathrm{~d}$ (Sts. 22-25). At these stages, peripheral projections of the most rostral eight segments consist of one to four individual neurites (Fig. 1). The numbers of peripheral projections on the injected and control sides of embryos were compared. XShal RNA overexpression leads to only a slight $(\sim 10 \%, p \leq 0.004$; Fig. 2$)$ reduction in peripheral neurite outgrowth in situ, in contrast with the large reduction in the number of morphologically differentiated neurons containing excess potassium channel RNA that appear in culture. The average percentage of embryos exhibiting the phenotype is $20 \pm 10 \%$ and ranges between $9 \%$ and $62 \%$ within individual clutches of embryos ( $n=4$ clutches).

To determine whether overexpression of XShal RNA alters projection number by reducing the number of neurons, HNKlabeled Rohon-Beard somata of Sts. 22-25 embryos were counted. The ratio of the number of labeled cells on the XSha1 RNAinjected side relative to the number on the control injected side is $1.1 \pm 0.1$ (mean $\pm \mathrm{SEM}, n=14$ embryos). If the entire population of HNK-labeled cells, which also includes interneurons, is considered, the ratio of the number of labeled cells on the XSha1 RNA-injected side relative to the number on the control injected side is still $1.1 \pm 0.1$ (mean \pm SEM, $n=26$ embryos).

In sum, these data indicate that XSha1 RNA overexpression in half of the embryo leads to only a slight reduction in situ in 


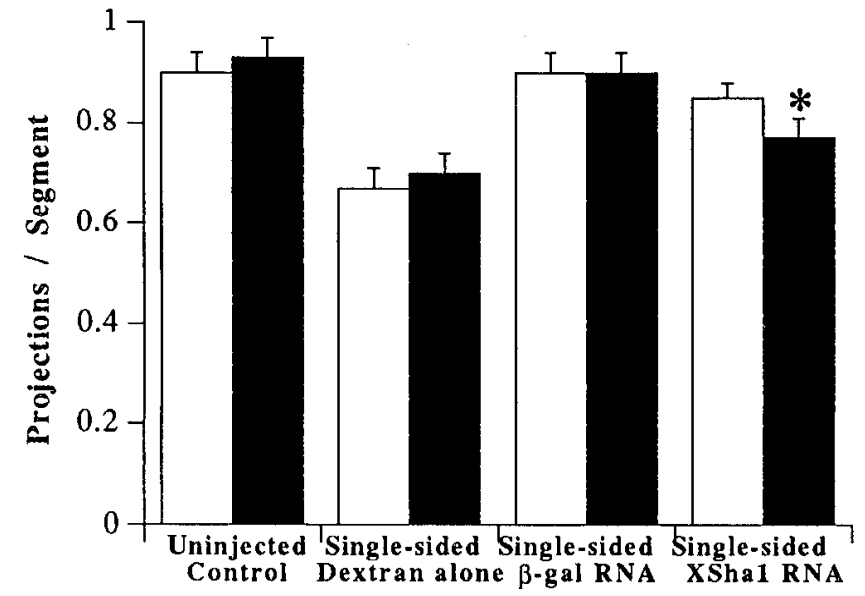

Figure 2. Overexpression of XSha1 but not $\beta$-galactosidase RNA in half of the embryo reduces the number of peripheral projections of Rohon-Beard neurons per segment. Embryos were injected into a single blastomere at the two-cell stage with the indicated RNAs and lineage tracers. Within an embryo, the numbers of projections per segment were compared between injected or left (filled bars) and uninjected or right (open bars) sides. Control uninjected embryos $(n=14)$ have approximately one projection per segment as do embryos that were injected into a single blastomere with $\beta$-galactosidase RNA $(n=13) ; 29$ embryos that had the fluorescent lineage tracer injected alone were examined. However, embryos that were injected in a single side with XShal RNA $(n=26)$ have a small $(\sim 10 \%)$ but significant $\left({ }^{*} p<0.004\right)$ reduction in the number of projections per scgment on the injected side. $\mathrm{xx}$ embryos injected with dextran were examined. Values are mean \pm SEM.

the number of peripheral projections of Rohon-Beard cells. Since this phenotype is much milder than the one present in vitro, endogenous factors that are present after St. 15 in vivo may rescue neurons from the dramatic effects observed in vitro.

\section{Morphological differentiation in vitro}

Cultures prepared from uninjected St. 15 embryos contain postmitotic primary neurons, which include Rohon-Beard, inter-, and motor neurons (Spitzer and Lamborghini, 1976; Lamborghini, 1980; Bixby and Spitzer, 1984; Lamborghini and Iles, 1985; Hartenstein, 1989). The reduced numbers of morphologically identifiable neurons observed after potassium channel overexpression in previous in vitro experiments (Jones and Ribera, 1994) could be due to delayed withdrawal from the mitotic cycle. Accordingly, the time at which neural tissue is removed from the embryo would influence the ability of neurons to differentiate morphologically in vitro. This prediction was tested by preparing cultures as usual from St. 15 embryos as well as older embryos (up to St. 25). Consistent with previous work, the number of morphologically differentiated neurons derived from the injected side is reduced significantly in cultures preparcd from St. 15 as well as St. 18 embryos (Fig. 3). However, there is an increase in the number of morphologically differentiated neurons derived from the injected side in cultures prepared from Sts. 19-25 embryos.

It is possible that the extended in vivo development diminishes functional overexpression of potassium current. Action potentials were recorded from neurons 2 to $4 \mathrm{hr}$ after plating cells dissuciated from St. 22 embryos. The durations of action potentials recorded from fluorescent neurons were 16-fold briefer than those recorded from nonfluorescent neurons (Table 1). This is consistent with previously observed shortening of the action po-

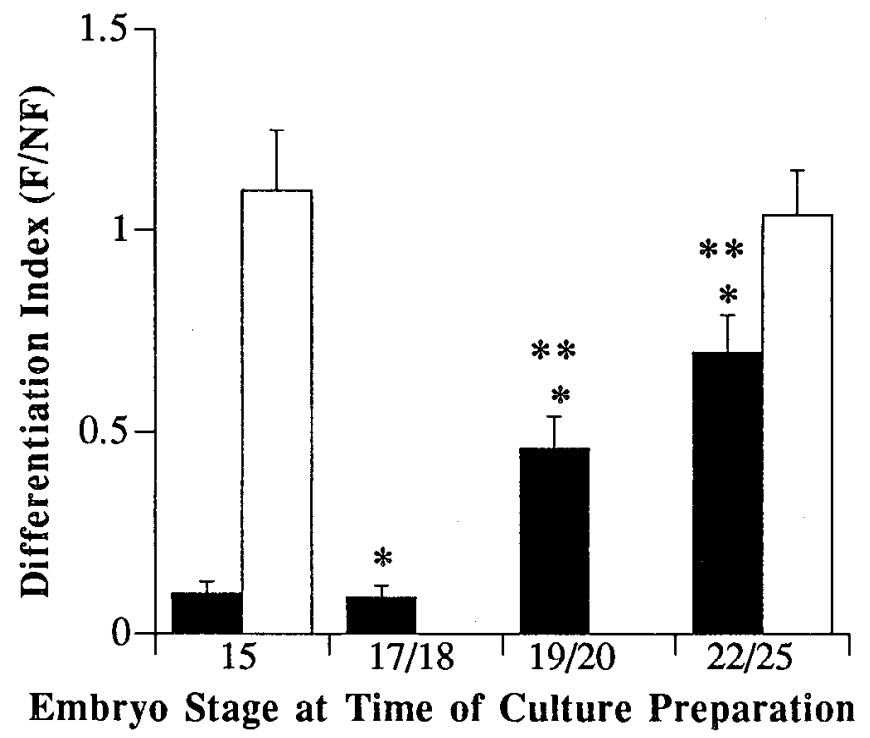

Figure 3. Differentiation index recovers in cultures prepared from older embryos. Embryos were injected into one cell at the two-cell stage with either control (open bar; tracer with or without $\beta$-galactosidase RNA) or XShal RNA (filled bar) solutions and allowed to develop to various stages: $15,17-18,19-20,22-25$. Cultures were prepared and neurons were scored at a time corresponding to $24 \mathrm{hr}$ after stage 15 . Development of the neural plate/tube in situ for an additional 3-8 hr (Sts. 19-25) promotes an increase in the differentiation index. Values are the mean $\pm \mathrm{SEM}$ of the ratio in 8-18 dishes for each condition. $* p<0.0001$ versus dextran control; ${ }^{*} p<0.0005$ versus XShal St. 15 .

tential duration in neurons overexpressing potassium channel RNA cultured from St. 15 embryos (Jones and Ribera, 1994). Further, the degree of shortening is that predicted for at minimum a threefold increase in potassium current density (Lockery and Spitzer, 1992). Direct examination of delayed rectifier potassium current $\left(I_{\mathrm{Kv}}\right)$ was done $12-24 \mathrm{hr}$ after plating. $I_{\mathrm{Kv}}$ density was larger in fluorescent versus nonfluorescent neurons (Table 1). These findings indicate that functional overexpression of potassium current RNA is still apparent in neurons cultured from older embryos.

The recovery in the number of morphologically identifiable neurons derived from the injected side at Sts. $20-22$ is consistent with a delay in the time at which neurons become postmitotic. Alternatively, interactions with or factors derived from the uninjected control side may mediate the observed compensation. To distinguish between these two possibilities, both cells of the two-cell embryo were injected with XShal RNA; additional growth in situ would still compensate for delayed exit from the cell cycle but not for interactions or factors derived from a normal uninjected side. To permit identification of neurons derived from each of the two injected cells, one cell was injected with RNA and rhodamine-conjugated dextran and the other with RNA and fluorescein-conjugated dextran. Injection of irrelevant RNA (i.e., $\beta$-galactosidase RNA) has no effect on the number of morphologically differentiated neurons (Fig. 4). The absolute number of morphologically differentiated neurons from either side is low in cultures prepared at St. 15 from embryos double injected with XShal RNA at the two-cell stage. When cultures are prepared from older XSha1 RNA double-injected embryos, the number of morphologically differentiated neurons increases but are substantially reduced compared to the number obtained from XShal RNA single injected embryos. These results suggest 
Table 1. Biophysical characteristics of neurons in cultures prepared from St. 22 embryos

\begin{tabular}{lllll} 
& Action potential & & $\begin{array}{l}\text { Delayed rectifier K current } \\
\text { density }\left(\mathrm{pA} / \mu \mathrm{m}^{2}\right) \\
\text { at }+25 \mathrm{mV} \mathrm{step}\end{array}$ & $n$ \\
\hline Nonfluorescent & $201 \pm 67$ & $n$ & $1.44 \pm 0.15$ & 12 \\
Fluorescent & $12 \pm 5^{*}$ & 10 & $2.28 \pm 0.69$ & 9 \\
\hline
\end{tabular}

Action potentials were recorded 2-4 hr after plating, and delayed rectifier potassium currents were recorded betwecn 12 and $18 \mathrm{hr}$ after plating, to make them comparable to times of these measurements in cultures prepared from St. 15 embryos (Jones and Ribera, 1994).

* Significantly different from nonfluorescent, $p<0.02$, Student's $t$ test (two Lailed).

that a delay in the exit from the cell cycle is not the relevant mechanism. Recovery apparently requires the absence of overexpressed XShal RNA in half of the embryo during Sts. 1822 .

These results raise the possibility that neurite outgrowth in situ may be more affected when XShal RNA overexpression occurs throughout the embryo. Embryos were injected at the two-cell stage into both blastomeres with either XShal or $\beta$-galactosidase RNA and dextran. However, the control ( $\beta$-galactosidase)-injected embryos appear to have a substantial reduction in the number of projections (compare Figs. 2, 5), suggesting that the double injection protocol itself may have an adverse effect in situ. From whole-mount analysis, it was not possible to detect a specific phenotype produced hy overexpressing RNA throughout the embryo. In additional experiments, embryos were injected with XShal RNA and dextran on one side and $\beta$-galactosidase RNA and dextran on the contralateral side; both sides show a reduction in projection number, although the XShal injected side appears to be more affected (Fig. 5).

\section{Effect of intracellular calcium buffers on morphological differentiation in vitro}

Between Sts. 20 and 25, spinal neurons generate long duration action potentials (Spitzer and Lamborghini, 1976; Baccaglini and Spitzer, 1977). Long duration impulses trigger transient elevations of intracellular calcium that are required for many aspects of neuronal differentiation ( $\mathrm{Ca}^{2+}$ spikes; Spitzer, 1994). Potassium channel overexpression, by reducing impulse duration, may suppress transicnt calcium clevations. To determine whether elimination of transient elevations of intracellular calcium reduces neuronal morphological differentiation, chelators of intracellular calcium were applied to cells in culture. The number of neurons or myocytes in treated dishes was expressed as the ratio of their number to the mean number in control dish-
A. XSha1 RNA Injections

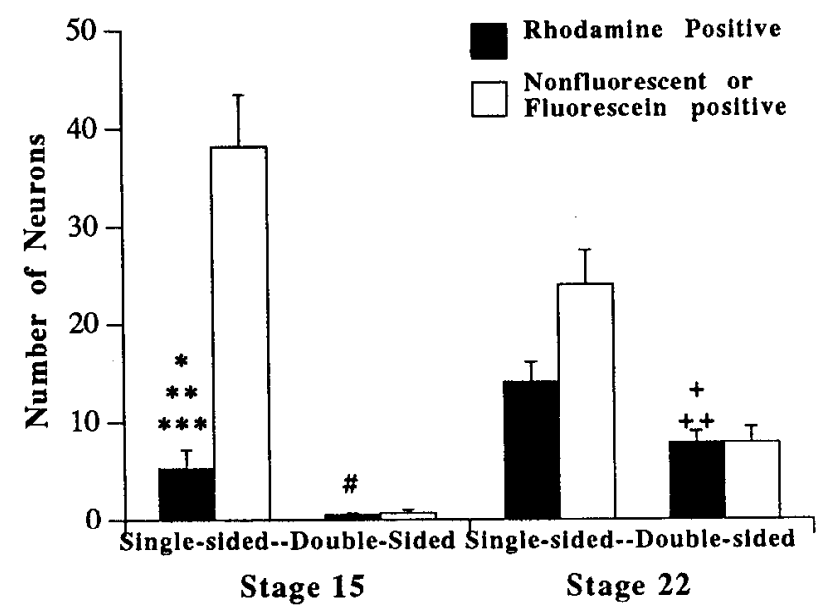

B.

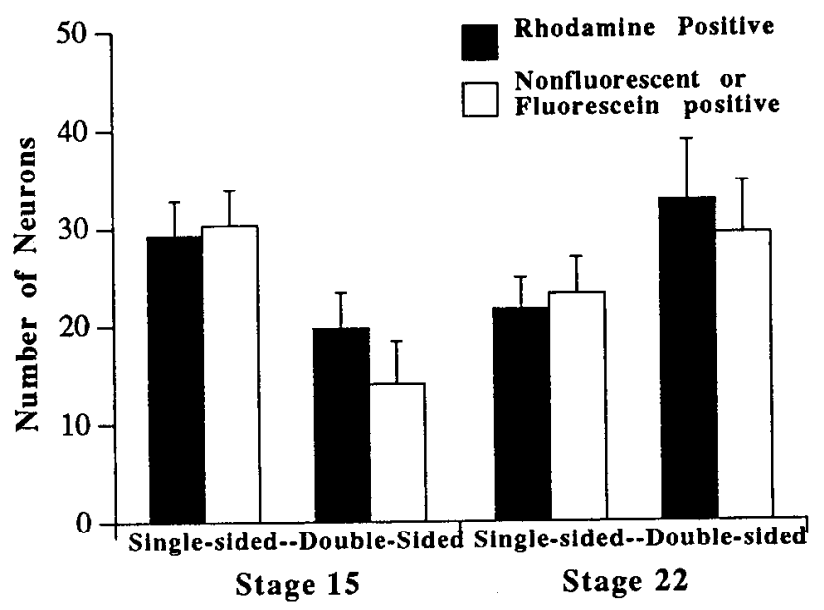

Figure 4. The increase in number of morphologically differentiated neurons in culture observed by culturing older embryos is not observed when cultures are prepared from embryos that were injected with XShal RNA in both hemispheres. A, Embryos were injected into either a single hemisphere (single-sided) or both hemispheres (double-sided) at the two-cell stage with XShal RNA and a fluorescent lineage tracer. Cultures were prepared from St. 15 or 22 embryos. The total number of morphologically differentiated neurons was scored at a time corresponding to 24 $\mathrm{hr}$ after stage 15. When embryos are injected into both hemispheres with XShal RNA, the total number of morphologically differentiated neurons derived from each injected side is low for both Sts. 15 and 22 cultures. Six comparisons yielded significant $p$-values: *, **, and *** are for XShal St. 15 single-sided fluorescent versus St. 15 single-sided nonfluorescent $(p<0.0001)$, St. 15 double-sided XShal $(p<0.03)$, and St. 22 singlesided XShal fluorescent $(p<0.003)$, respectively; \# is for double-sided XShal St. 15 versus St. $22(p<0.0001)$; + and ++ are for XSha1 single-sided St. 22 versus St. 22 nonfluorescent $(p<0.02)$ and St. 22 double-sided XSha1 $(p<0.02)$, respectively. $B$, Embryos were injected into either a single hemisphere (single-sided) or both hemispheres (double-sided) at the two-cell stage with $\beta$-galactosidase RNA and a fluorescent lineage tracer. Cultures were prepared from St. 15 or 22 embryos. The total number of morphologically differentiated neurons was scored at a time corresponding to $24 \mathrm{hr}$ after Stage 15 . Injection of tracer or $\beta$-galactosidase RNA does not affect the number of morphologically differentiated neurons that appear in culture, regardless of the stage of culture preparation or whether injections are single- or double-sided. No comparison yielded a $p$-value $\leq 0.05$. For both $A$ and $B$, the data for single-sided injections are obtained from the experiments in Figure 3 , where they are expressed in terms of a differentiation index. Values represent the mean $( \pm S E M)$ of $7-29$ dishes for each condition. 


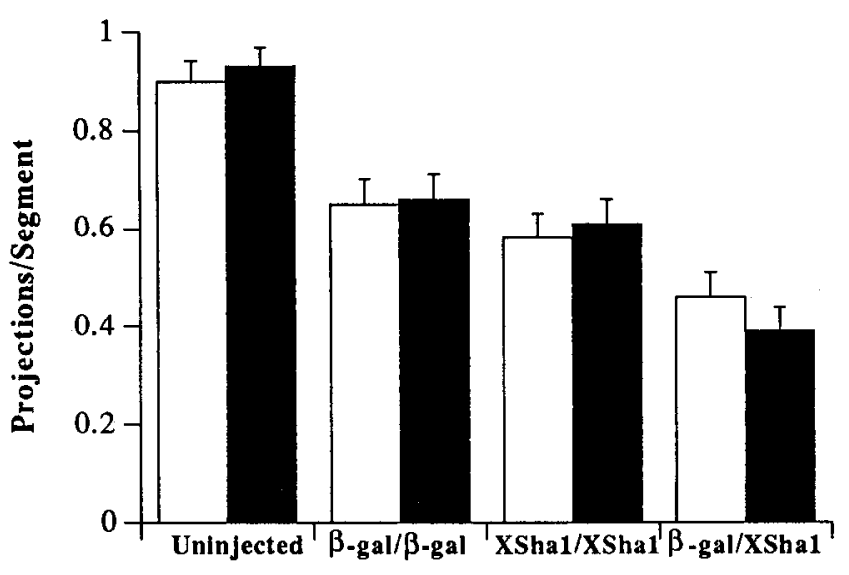

Figure 5. The number of peripheral projections per segment is similar on both sides when overexpression of XShal RNA occurs throughout the embryo. Embryos were injected into both blastomeres at the twocell stage with the indicated RNAs and lineage tracers (see Materials and Methods). Within an embryo, the numbers of projections per segment were compared between right or $\beta$-galactosidase RNA (open bars) injected and left or XSha1 RNA ( filled bars) injected sides. Note, however, that in comparison to Figure 2, the number of projections per segment seems to be reduced by injection of control ( $\beta$-galactosidase) RNA into both hemispheres. The number of injected embryos examined ranged between 26 and 43 . Values are mean \pm SEM.

es; overall, the mean number of neurons and myocytes in control dishes was set at $100 \%$. The normalized number of morphologically differentiated ncurons in cultures loaded with $0.75-1 \mu \mathrm{M}$ DM-BAPTA-AM is reduced. In contrast, loading of $5 \mu \mathrm{M}$ EGTA-AM has no effect on the number of morpholngically differentiated neurons (Fig. 6). BAPTA binds $\mathrm{Ca}^{2+}$ more quickly than does EGTA, and, thus, would be expected to bind $\mathrm{Ca}^{2+}$ fast enough to prevent effects due to rapid intracellular $\mathrm{Ca}^{2+}$ elevations (e.g., Adler et al., 1991; Robitaille et al., 1993). The effect of BAPTA is unlikely to be due to toxicity, since it is effective only when applied before the period of calcium sensitivity (Fig. 6; Holliday et al., 1991). Further, at these concentrations, the chelators have no significant effect on the numbers of morphologically differentiated myocytes (Fig. 6). The early and selective effect of DM-BAPTA-AM on the number of morphologically differentiated neurons suggests that intracellular calcium transients are involved in triggering morphological differentiation of neurons. These findings support the possibility that XSha1 RNA overexpression perturbs morphological differentiation of neurons by reducing action potential durations and suppressing calcium transients.

\section{Effects of intracellular calcium buffers and electrical activity on recovery of in vitro differentiation index promoted by extended development in vivo}

It is possible that factors or interactions that promote morphological differentiation are provided by action potential generation in or cellular interactions with the normal uninjected side. Further, these factors and interactions could then be available to the uninjected side in embryos in which XShal RNA is overexpressed in only one side. For example, electrical coupling is present in the developing neural tube until St. 25 (Spitzer, 1982) and may compensate for the suppression of long-duration impulses on the side of the neural tube containing exogenous XSha1 RNA.

To examine whether action potential generation provides such

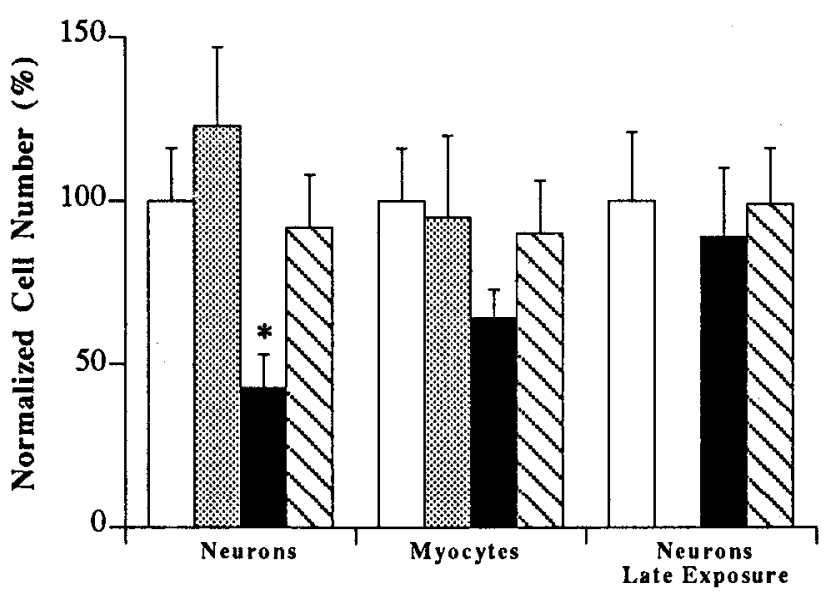

Figure 6. Treatments that rapidly buffer intracellular calcium transients lead to a reduction in the number of neurons that differentiate morphologically in culture. For these experiments, cultures were prepared from St. 15 uninjected embryos. Cells in culture were loaded with either BAPTA-AM or EGTA-AM for 1 hr shortly after plating. The number of morphologically differentiated neurons (left) and myocytes (middle) were counted at $24 \mathrm{hr}$ after plating. The number of neurons or myocytes in treated dishes was expressed as the ratio of their number to the mean number in control dishes; overall, the mean number of neurons and myocytes in control dishes was set at 100\%. BAPTA $(1 \mu \mathrm{M})$ (filled bars) reduces the number of morphologically differentiated neurons ( $p<0.002$ ); $0.5 \mu \mathrm{M}$ BAPTA (stippled bars) did not produce a significant effect. Although there is an apparent reduction in the number of morphologically differentiated myocytes, the result is not statistically significant $(p<0.64)$. EGTA $(5 \mu \mathrm{M})$ (hatched bars) was without effect, suggesting that transient rather than steady state eleva tions in intracellular calcium are required for morphological differentiation of neurons. In some experiments, cells were loaded with BAPTA at $12 \mathrm{hr}$, rather than $1 \mathrm{hr}$, after plating (Neurons, Late Exposure); morphological differentiation of neurons was not affected, indicating that the effect of BAPTA is not due to nonspecific toxicity. The number of morphologically differentiated neurons or myocytes in each experimentally treated culture was calculated as a ratio of the mean number of morphologically differentiated neurons or myocytes, respectively, overall. Values represent the mean ( \pm SEM) of $4-15$ dishes for each condition.

signals, neurons in situ were exposed to the sodium channel blocker, TTX, between Sts. 17 and 22 . TTX reduces by $\sim 80 \%$ spontaneous action potential generation that is evidenced by calcium imaging (Gu et al., 1994). TTX was applied to live embryos by injection into the archenteron. Exposure to TTX does not prevent the increase in differentiation index achieved by culturing embryos at St. 22 (Fig. 7). Thus, TTX sensitive sodium channels do not appear to be involved in mediating recovery of the differentiation index by prolonged development in situ.

Since TTX reduces action potential firing by only $80 \%$, it is possible that calcium channel activity is sufficient to maintain adequate impulse generation. This possibility was tested by cxposure to $\omega$-conotoxin GVIA, an efficient blocker of calcium current in Xenopus spinal neurons (Gu and Spitzer, 1993). However, blocking concentrations of $\omega$-conotoxin do not prevent the recovery of the differentiation index (Fig. 7).

To examine whether intracellular calcium transients provide compensatory factors, neurons in situ were exposed to the intracellular calcium chelators, EGTA-AM and BAPTA-AM. Exposure to BAPTA, but not to EGTA, does prevent the increase in differentiation index achieved by culturing embryos at St. 22 (Fig. $7 ; p<0.001$ ). BAPTA is more effective than EGTA, sug- 


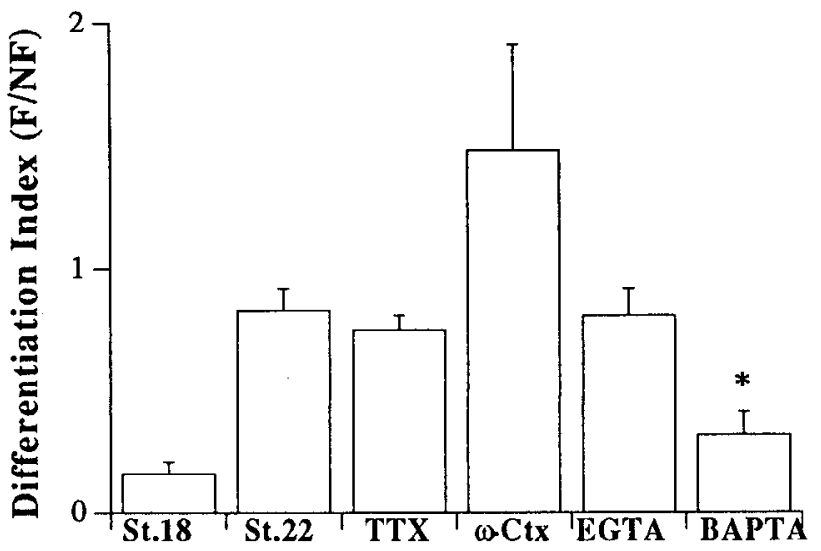

Figure 7. Suppression of calcium transients but not electrical activity prevents the increase in differentiation index promoted by extended development in situ. Embryos were injected at the two-cell stage in a single blastomere with XSha1 RNA and rhodamine-conjugated dextran. TTX, $\omega$-conotoxin, EGTA, or BAPTA $(\sim 1 \mu \mathrm{M}$; see Materials and Methods) were injected into the archenteron of developing embryos at either St. 17 or St. 19. At the equivalent of St. 22, injected embryos were prepared for culture as were control Sts. 15-18 embryos. The differentiation index was specifically reduced by exposure to BAPTA $(p<$ 0.0013 ), suggesting that intracellular calcium transients mediate the recovery of differentiation index that is promoted by additional development in situ. Although there is an apparent increase in the differentiation index upon exposure to $\omega$-conotoxin, the result is not statistically significant $(p<0.18)$. Values represent the mean $(+$ SEM $)$ of $6-26$ dishes for each condition from three to five culture runs.

gesting that rapid elevations in intracellular calcium mediate in vivo recovery.

\section{Discussion}

The major finding of this work is that overexpression of potassium channel RNA affects morphological differentiation of neurons differently in vitro than it does in vivo. The results are consistent with the hypothesis that interactions and/or factors are present in vivo that compensate for the effects of potassium channel RNA overexpression. For example, in cultures that are prepared from older embryos, there is an increase in the number of morphologically differentiated neurons containing excess potassium channel RNA. Further, the ability of BAPTA-AM, but not EGTA-AM, to inhibit the rescue effect when injected into neural plate stage XSha1 RNA-containing embryos suggests a role for intracellular calcium transients in the relevant mechanism.

A possible limitation to this conclusion is that only one class of primary spinal neurons, Rohon-Beard cells, was examined in detail in vivo. This population was selected for analysis because their identification in situ is unambiguous. In contrast, identification of primary motor- or interneurons is complicated by the presence of more numerous secondary motor- and interneurons. It is possible that primary motor- or interneurons in situ may be more affected than Rohon-Beard cells. However, each of these three classes of primary neurons contributes roughly equally to the population that differentiates in vitro (Bixby and Spitzer, 1984). If Rohon-Beard neurons are resistant to the effects of potassium channel RNA overexpression in vivo and in vitro, the differentiation index would be expected to reach values of not less than $\sim 0.33$. In contrast, the average value was substantially lower (0.1; e.g., Fig. 3).

Another consideration is that the times of examination of mor- phological differentiation in vivo and in vitro are not the same. Only postmitotic primary neurons differentiate morphologically in culture (Spitzer and Lamborghini, 1976). Maximum morphological differentiation in vitro occurs $\sim 24$ hr after plating, which corresponds to a $2 \mathrm{~d}$ (St. 34) rather than a $1 \mathrm{~d}$ (Sts. 22-25) embryo. In order to enable examination of the same neuronal population in vivo and in vitro, Sts. 22-25 embryos were examined at the whole-mount level, because HNK labeling clearly reveals individual Rohon-Beard neurons at these stages. At $2 \mathrm{~d}$ in situ, secondary neurons are the major population of morphologically differentiated neurons in vivo (Hartenstein, 1989). In initial experiments, cultures were also examined at $6 \mathrm{hr}$ after plating which corresponds to Sts. 22-25 embryos. Although fewer morphologically differentiated neurons were present at this time ( $<10$ vs. 60 neurons at 6 and $24 \mathrm{hr}$ after plating, respectively; Ribera and Spitzer, 1989), differentiation indexes were similar to those at $24 \mathrm{hr}$ (data not shown).

Interpretation of experiments using the AM forms of BAPTA and EGTA requires the usual cautions regarding efficiency of trapping, partitioning, and stability. The AM forms of the calcium indicator dyes, Fura-2 and Fluo-3, have been used extensively to monitor intracellular calcium levels in Xenopus spinal neurons both in vivo and in vitro (Holliday and Spitzer, 1990; Barish, 1991; Holliday et al., 1991; Gu and Spitzer, 1993; Gu et al., 1994). Sequestration of the compounds into intracellular compartments did not appear to affect measurement, since similar patterns of calcium signals were detected in regions of the cytoplasm that were either rich or poor in yolk granules (Barish, 1991). Further, loading of neurons with the AM forms of Fura-2 and Fluo-3 did not affect cell survival (Gu et al., 1994). After loading and washing, imaging is possible for a minimum of 2 $\mathrm{hr}$, indicating that these compounds are partitioned and stable in appropriate compartments for at least this duration. In the present studies, BAPTA-AM or EGTA-AM were injected into embryos at St. 18 and cultures were prepared approximately $4 \mathrm{hr}$ later at St. 22. Given the above considerations, it may be expected that BAPTA and EGTA were effective intracellular chelators for a minimum period of $2 \mathrm{hr}$. It is not known whether repeated injections of BAPTA-AM or EGTA- AM would have produced larger or different results. The ability of BAPTA-AM but EGTA-AM to prevent the rescue effect supports the view that BAPTA's actions are due to suppression of intracellular calcium transients rather than a nonspecific toxic action such as formation of formaldehyde.

The extent to which the mechanism underlying the rescue effect relates to the mechanism by which potassium channel RNA overexpression inhibits morphological differentiation of neurons is not known. It is possible that the rescue effect is achieved in vivo by directly inhibiting potassium channel overexpression. However, functional overexpression at the level of the plasma membrane was still apparent. Additional possibilities include that (1) potassium channel overexpression interferes intracellularly with the normal signal transduction involved in morphological differentiation (Holliday and Spitzer, 1990; Holliday et al., 1991; Gu et al., 1994) and/or (2) the rescue effect is achieved by activating an independent and different signaling pathway that promotes morphological differentiation.

The data presented here establish the presence of an in vivo rescue effect that is not due to a recovery from cell death or delayed differentiation. Further, substantial rescue requires that part of the embryo have normal levels of potassium channel RNA (i.e., no overexpression). During the period of extended 
development in vivo (Sts. 15-22), spontaneous intracellular calcium transients occur that influence morphological differentiation (Gu et al., 1994). Action potential generation triggers a kinetically rapid elevation of intracellular calcium referred to as a calcium spike (Gu et al., 1994). In our experiments, it is possible that action potential generation occurs normally in the uninjected side of the embryo, and that membrane depolarization is communicated to the injected side of the embryo via gap junctions. Surprisingly, neither TTX nor $\omega$-conotoxin is able to suppress the rescue effect observed by prolonged development in vivo (Fig. 7). Perhaps, under these conditions, subthreshold levels of membrane depolarization continue to occur, are communicated via gap junctions, and are sufficient to provoke sufficient release of calcium from intracellular stores. In fact, release of calcium from intracellular stores appears to have a much higher sensitivity to calcium ion at these early stages of neuronal differentiation than is apparent $24 \mathrm{hr}$ later (Holliday et al., 1991). Further, kinetically slow elevations of intracellular calcium ("waves"; Gu et al., 1994) occur during this period independently of action potential generation. Alternatively, in the uninjected side of the embryo a factor may be secreted without generation of impulses; this factor may promote morphological differentiation and diffuse to the injected side and mediate the recovery effect. Such an explanation may account for the observation that minimal to no rescue was observed when the entire neural plate was removed and cultured as an explant; under these conditions, the concentration of such a putative secreted factor would be reduced by the relatively large volume of the culture dish medium.

These studies indicate that in vivo analysis of the effects of over- or underexpression may be misleading, since there is the possibility of additional mechanisms that diminish or abolish the direct consequences of the misexpression. In the face of abnormal gene expression, cells may develop normally, not because the misexpressed gene products are without effect, but because they provoke compensatory reactions in the host.

\section{References}

Adler EM, Augustine GJ, Duffy SN, Charlton MP (1991) Alien intracellular calcium chelators attenuate neurotransmitter release at the squid giant synapse. J Neurosci 11:1496-1507.

Amigorena S, Choquet D, Teillaud J-L, Korn H, Fridman WH (1990) Ion channel blockers inhibit B cell activation at a precise stage of the Gl phase of the cell cycle. J Immunol 144:2038-2045.

Anglister L, Farber IC, Shahar A, Grinvald A (1982) Localization of voltage-sensitive calcium channels along developing neurites: their possible role in regulating neurite elongation. Dev Biol 94:351-365.

Baccaglini PI, Spitzer NC (1977) Developmental changes in the inward current of the action potential of Rohon-Beard neurones. J Physiol (Lond) 271:229-250.

Barish ME (1991) Increases in intracellular calcium ion concentration during depolarization of cultured embryonic Xenopus spinal neurones. J Physiol (Lond) 444:545-565.

Bixby JL, Spitzer NC (1984) The appearance and development of neurotransmitter sensitivity in Xenopus embryonic spinal neurones in vitro. J Physiol (Lond) 353:143-155.

Blair LAC (1983) The timing of protein synthesis required for the development of the sodium action potential in embryonic spinal neurons. J Neurosci 3:1430-1436.

Choquet D, Sarthou P, Primi D, Cazenave P-A, Korn H (1987) Modulation of voltage-dependent potassium channels in B lymphocytes. Biochem Pharmacol 37:3797-3802.

Cohan CS (1992) Depolarization-induced changes in neurite elongation and intracellular $\mathrm{Ca}^{2+}$ in isolated Helisoma neurons. J Neurobiol 23: 983-996.

Cohan CS, Connor JA, Kater SB (1987) Electrically and chemically mediated increases in intracellular calcium in neuronal growth cones. J Neurosci 7:3588-3599.

Collins F, Schmidt MF, Guthrie PB, Kater SB (1991) Sustained increase in intracellular calcium promotes neuronal survival. J Neurosci. 11: $2582-2587$.

Davenport RW, Kater SB (1992) Local increases in intracellular calcium elicit local filopodial responses in helisoma neuronal growth cones. Neuron 9:405-415.

Day ML, Pickering SJ, Johnson MH, Cook DI (1993) Cell-cycle control of a large-conductance $\mathrm{K}^{+}$channel in mouse early embryos. Nature 365:560-562.

$\mathrm{Gu}$ X, Spitzer NC (1993) Low-threshold $\mathrm{Ca}^{2+}$ current and its role in spontaneous elevations of intracellular $\mathrm{Ca}^{2+}$ in developing Xenopus neurons. J Neurosci 13:4936-4948.

Gu X, Olson EC, Spitzer NC (1994) Spontaneous neuronal calcium spikes and waves during early differentiation. $J$ Neurosci, in press.

Hamill OP, Marty A, Neher E, Sakmann B, Sigworth FJ (1981) Improved patch-clamp techniques for high resolution current recording from cells and cell-free membrane patches. Pfluegers Arch 391:85100.

Hartenstein V (1989) Early neurogenesis in Xenopus: the spatio-temporal pattern of proliferation and cell lineages in the embryonic spinal cord. Neuron 3:399-411.

Hartenstein V (1993) Early pattern of neuronal differentiation in the Xenopus embryonic brainstem and spinal cord. J Comp Neurol 328: 213-231.

Holliday J, Spitzer NC (1990) Spontaneous calcium influx and its roles in differentiation of spinal neurons in culture. Dev Biol 141:13-23.

Holliday J, Adams RJ, Scjnowski TJ, Spitzer NC (1991) Calciuminduced release of calcium regulates differentiation of cultured spinal neurons. Neuron 7:787-796.

Jones SM, Ribera AB (1994) Overexpression of a potassium channel gene perturbs neural differentiation. J Neurosci 14:2789-2799.

Klymkowsky MW, Hanken J (1991) Whole-mount staining of Xenopus and other vertebrates. In: Methods in cell biology (Kay BK, Peng HB, eds), pp 409-411. New York. Academic.

Lamborghini JE (1980) Rohon-Beard cells and other large neurons in Xenopus embryos originate during gastrulation. J Comp Neurol 189: 323-333.

Lamborghini JE, Iles A (1985) Development of a high-affinity GABA uptake system in embryonic amphibian spinal neurons. Dev Biol 112: $167-176$.

Lockery SR, Spitzer NC (1992) Reconstruction of action potential development from whole-cell currents of differentiating spinal neurons. J Neurosci 12:2268-2287.

Mattson MP, Kater SB (1987) Calcium regulation of neurite elongation and growth cone motility. J Neurosci 7:4034-4043.

Moon RT, Christian J (1989) Microinjection and expression of synthetic mRNAs in Xenopus cmbryos. Technique 1:76-89.

Nieuwkoop PD, Faber J (1967) Normal table of Xenopus laevis. Amsterdam: North Holland.

Nordlander RH (1989) HNK-1 marks earliest axonal outgrowth in Xenopus. Dev Brain Res 50:147-153.

Ribera AB, Spitzer NC (1989) A critical period of transcription required for differentiation of the action potential of spinal neurons. Neuron 2:1055-1062.

Robitaille R, Garcia ML, Kaczorowski GJ, Charlton MP (1993) Functional colocalization of calcium and calcium-gated potassium channels in control of transmitter release. Neuron 11:645-655.

Sakmann B, Neher E (1983) Geometric parameters of pipettes and membrane patches. In: Single-channel recording (Sakmann B, Neher E, eds), pp 37-51. New York: Plenum.

Spitzer NC (1982) Voltage and stage-dependent uncoupling of RohonBeard neurones during embryonic development of Xenopus tadpoles. J Physiol (Lond) 330:145-152.

Spitzer NC (1994) Spontaneous $\mathrm{Ca}^{2+}$ spikes and waves in embryonic neurons: signaling systems for differentiation. Trends Neurosci 17: $115-118$.

Spitzer NC, Lamborghini JE (1976) The development of the action potential mechanism of amphibian neurons isolated in cell culture. Proc Natl Acad Sci USA 73:1641-1645.

Taylor JSH, Robcrts A (1983) The early development of the primary sensory neurones in an amphibian embryo: a scanning electron microscope study. J Embryol Exp Morphol 75:49-66.

Tsien RY (1981) A non-disruptive technique for loading calcium buffers and indicators into cells. Nature 290:527-528. 